\title{
Intrinsic, Extrinsic, and the Constitutive A Priori
}

\author{
László E. Szabó ${ }^{1}$ (I) \\ Received: 2 January 2019 / Accepted: 21 June 2019 / Published online: 15 July 2019 \\ (c) The Author(s) 2019
}

\begin{abstract}
On the basis of what I call physico-formalist philosophy of mathematics, I will develop an amended account of the Kantian-Reichenbachian conception of constitutive a priori. It will be shown that the features (attributes, qualities, properties) attributed to a real object are not possessed by the object as a "thing-in-itself"; they require a physical theory by means of which these features are constituted. It will be seen that the existence of such a physical theory implies that a physical object can possess a property only if other contingently existing physical objects exist; therefore, the intrinsic-extrinsic distinction is flawed.
\end{abstract}

Keywords Reichenbach · Constitutive a priori · Physicalism · Formalism · Meaning · Truth $\cdot$ Holism $\cdot$ intrinsic property

\section{Introduction}

1. There is a long-standing debate in contemporary metaphysics about the precise definition of intrinsic property. The great majority of the suggested definitions are some enhanced version of Jaegwon Kim's (1982) definition, expressing the following simple idea:

Intrinsic properties are the properties a particular object would have even if no other contingently existing objects existed in the world. [1]

Although Kim's definition has been widely accepted as basically adequate criterion of intrinsicality, it has been challenged by subtle examples and amended at several points. Beyond these improvements, the intrinsic-extrinsic distinction is often discussed in a wider context of other closely related metaphysical problems, such as categoricaldispositional, pure-impure, relational-non-relational, interior-exterior distinctions;

László E. Szabó

laszlo.e.szabo@gmail.com

1 Department of Logic, Institute of Philosophy, Eötvös Loránd University Budapest, Múzeum krt 4/i, Budapest 1088, Hungary 
the problem of identity, duplicate, persistence; or the problem of Cambridge change (e.g., $[11,16,18-22,27,32])$. It is not my intention, however, to review these debates, as the aim of this paper is to challenge the concept of intrinsicality from a completely different point of view and to show that the intrinsic-extrinsic distinction is flawed on a more fundamental level.

2. Accordingly, my analysis will be restricted in two significant senses:

(a) The discussion will be restricted to the case of ordinary physical properties of physical objects.

(b) The whole analysis will remain within a radical physicalist ontological doctrine: The world can be completely accounted for by assuming that only physical entities exist.

\section{Constitutive A Priori}

3. An object can possess a property $X$ only if the term $X$ has meaning. This might sound too radical. Probably, a less radical statement would be easily accepted: The knowledge claim that an object possesses a property $X$ is possible only if the term $X$ has meaning. But, just here is the philosophically relevant point: this is not simply a matter of semantics. As Reichenbach pointed out in his Relativity Theory and A Priori Knowledge (1920) [25], the coordinative definitions, that is, the semantic conventions by which physical quantities or conceptions are defined in empirical terms, play constitutive role; they are constitutive a priori. "They define the individual elements of reality and in this sense constitute the real object." [25, p. 53]

Reichenbach sharply distinguishes two different aspects of Kantian a priori:

Kant's concept of a priori has two different meanings. First, it means "necessarily true" or "true for all times," and secondly, "constituting the concept of object." The second meaning must be clarified. According to Kant, the object of knowledge, the thing of appearance, is not immediately given. Perceptions do not give the object, only the material of which it is constructed. Such constructions are achieved by an act of judgment. The judgment is the synthesis constructing the object from the manifold of the perception. For this purpose it orders the perceptions according to a certain schema; depending on the choice of the schema, either an object or a certain type of relation will result. Intuition is the form in which perceptions present the material-thus performing another synthesis. But the conceptual schema, the category, creates the object; the object of science is therefore not a "thing-in-itself" but a reference structure based on intuition and constituted by categories. [25, pp. 48-49]

4. Let me give an example. Electrodynamics begins with the operational definitions of the basic electrodynamic quantities. For example, electric field strength is defined as the force felt by the unit test charge in electromagnetic field, when the test charge is at rest. ${ }^{1}$ This operational definition, therefore the notion of physical quantity called

\footnotetext{
${ }^{1}$ For the sake of simplicity I use this naive definition. For more precise operational definitions of electrodynamic quantities, see [13].
} 
electric field strength is a priori in the sense that it is prior to our empirical knowledge about the electromagnetic field. There are no empirical facts which would determine the convention by which this physical quantity is defined. In other words, the electromagnetic field, as a "thing-in-itself" does not determine that such a physical quantity is necessarily introduced for its characterization, as one of its fundamental feature. (In fact, the so called "displacement vector", a different physical quantity having a completely different operational definition, is an equally good alternative for the description of electromagnetic field.)

Once electric field strength is introduced, we can observe empirical facts about it. We can measure, for example, the electric field strength around a point charge being at rest, and, by inductive generalization, we can ascertain the Coulomb Law: $E=\frac{k Q}{r^{2}}$. To be sure, the law of physics we ascertained is a posteriori; it can be known only from empirical observations. But the features of the physical reality that the physical law talks about is a priori constructed by us.

5. All this means that the features (attributes, qualities, properties) attributed to a real object are not possessed by the object as a "thing-in-itself"; they require the existence of something else, an epistemic agent constituting them. This recognition outlines an argument against intrinsic-extrinsic distinction.

6. However, the Kantian-Reichenbachian account raises several problems. It is not clear at all what kind of ontological entity is involved by the required epistemic agency. Is it a contingently existing flesh and blood physical being? Or is it an abstract entity, as sometimes the Kantian "transcendental subject" is interpreted? [9, p. 53] To what extend is a semantic convention free? Are there "coordinating principles", "prescriptions for the conceptual side of the coordination", as Reichenbach [25, p. 54] presupposes? And what is the origin of such principles? Logic and mathematics? Are logical and mathematical truths necessary truths? If so, how to avoid then necessarily true synthetic a priori statements about the physical world? Finally, how to incorporate semantic and epistemological holism, the fact that "our statements about the external world face the tribunal of sense experience not individually but only as a corporate body" [24]?

In the next sections, I will attempt to formulate the argument outlined in point $\mathbf{5}$, in such a way that the above mentioned problems will be resolved or avoided. To achieve this aim, however, I need to redraw a larger picture.

\section{Physico-formalist Account of Logic and Mathematics}

7. I begin with a physicalist account of logic and mathematics. How can the logical and mathematical facts be accommodated in a purely physical ontology? Physicalism denies the existence of mental and abstract entities; consequently, there is no room left for any kind of platonism or mentalism in the philosophy of logic and mathematics. Therefore, two possibilities are left: a Millian-style physical realist approach, and formalism. We can disregard from the Millian realism, in which mathematics itself becomes a physical theory, in the sense of physical theory as described in the next section. So, formalism - more precisely, what I will call physico-formalism—seems to 
be the only account for logic and mathematics that can be compatible with physicalism. Therefore, the formalist approach is our starting point.

First we need to clarify: What are the logical/mathematical facts? The formalist thesis can be summarized in one famous sentence of Hilbert: "Mathematics is a game played according to certain simple rules with meaningless marks." [6, p. 38] Accordingly, in the formalist account, a mathematical statement/fact/truth is like " $\Sigma \vdash A$ ". According to the formalist view, neither $A$ nor the elements of $\Sigma$ are statements, which could be true or false. They are just meaningless strings, formulas of the formal system in question. The entailment $\vdash$ relation has nothing to do with "truth preserving if-then-type reasoning"; it simply stands for the fact that there is a finite sequence of meaningless strings of symbols, fitting into some structural patterns called "derivation rules". As a visual illustration, Fig. 1 shows a short example: the first order axiomatic formulation of group theory and the proof that $p(e, p(e, e))=e$ is a theorem; specifically, the sequence of formulas (1)-(9) constitutes a proof.

8. How can a formal system be accommodated in a purely physical ontology? Where are the states of affairs located in the physical world that make propositions like " $\Sigma \vdash A$ " true or false? For example, what are the facts of the physical world that make the sequence of formulas (1)-(9) a proof of $p(e, p(e, e))=e$ in Fig 1 ? Consider the first formula (1) in the proof. From ontological point of view, what does it mean that it is nothing else but axiom (G2)? It means that the formula $p(e, x)=x$ in row (1) is the same as the formula $p(e, x)=x$ in row (G2). More exactly: it means the physical fact that the arrangement of black and white pixels on the screen of my laptop on which I am writing this paper-or the arrangement of ink particles on the printout-in row (1) and the corresponding arrangement of black and white pixels in row (G2) are similar (congruent). Similarly, what does it mean that formula $\forall x p(e, x)=x$ in (2) is obtained from $p(e, x)=x$ by the derivation rule $(\mathrm{G})$ ? It means that replacing the pixel-configuration $\phi$ in $\phi \vdash \forall x \phi$, with copy/paste, by $p(e, x)=x$ in (1), the resulted pixel-configuration is $p(e, x)=x \vdash \forall x p(e, x)=x$, such that the left hand side is similar to the pixel-configuration $p(e, x)=x$ in (1) and the right hand side is similar to $\forall x p(e, x)=x$ in (2). And so on and so forth.

Of course, a formal system can be thought of in different physical forms; written with ink on paper, in the form of brain states and brain processes, in the form of electronic states and electronic processes in a computer, etc. What is important is the following observation:

The physico-formalist thesis The logical and mathematical facts, since being formal facts, are nothing but physical facts of physically existing formal systems consisting of signs and derivation patterns embodied in concrete physical objects, concrete physical configurations, and concrete physical processes. [2830]

The physico-formalist account remains completely within the physicalist ontology: there is no need for hypostatized entities-abstract, conceptual, or mental formal systems - which exist over and above the physically existing formal systems embodied in concrete physical objects, concrete physical configurations, and concrete physical processes. 


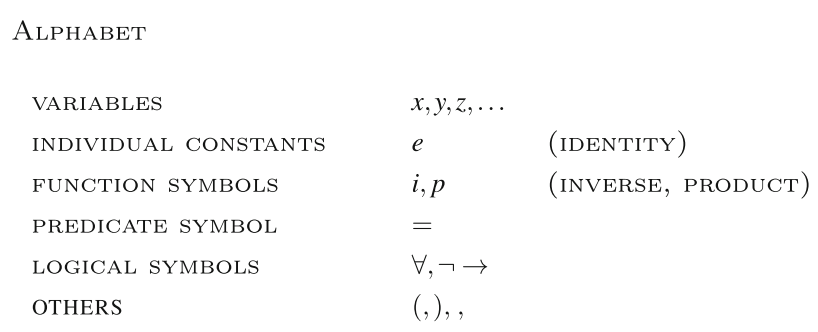

Derivation RUles
$(\mathrm{MP}) \quad\{\phi,(\phi \rightarrow \psi)\} \vdash \psi$
(MODUS PONENS)
(G) $\quad \phi \vdash \forall x \phi$
(GENERALIZATION)

\section{Axioms}

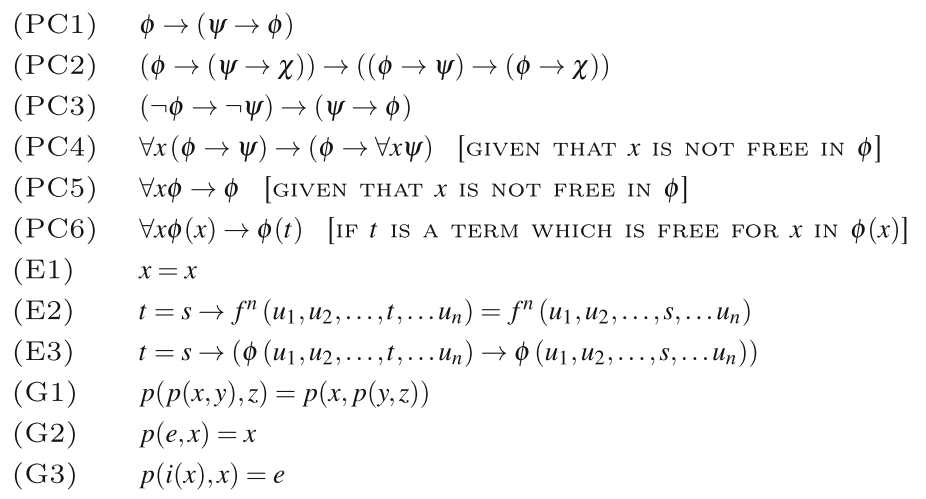

Theorem: $\quad p(e, p(e, e))=e$

\section{Proof:}
(1) $p(e, x)=x$
(2) $\forall x p(e, x)=x$
(3) $\forall x p(e, x)=x \rightarrow p(e, e)=e$
(4) $p(e, e)=e$
(5) $\forall x p(e, x)=x \rightarrow p(e, p(e, e))=p(e, e)$
(6) $p(e, p(e, e))=p(e, e)$
(2), (5), (MP)
(7) $\quad p(e, e)=e \rightarrow(p(e, p(e, e))=p(e, e) \rightarrow p(e, p(e, e))=e)$
(8) $\quad p(e, p(e, e))=p(e, e) \rightarrow p(e, p(e, e))=e$
(9) $\quad p(e, p(e, e))=e$
(6), (8), (MP)

Fig. 1 Group theory and one of its theorems 


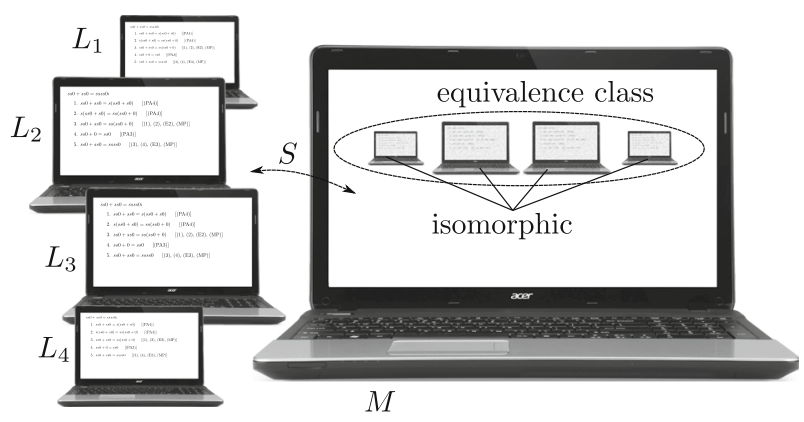

Fig. 2 In order to isolate the common essential features of different physically existing formal systems $L_{1}, L_{2}, L_{3}, \ldots L_{n}$ we must have a "meta-theory" of $L_{1}, L_{2}, L_{3}, \ldots L_{n}$, using another physically existing formal system $M$.

9. "Abstract" or "mathematical" formal system is often conceived as an entity obtained by abstraction, by isolating the common features in a number of particular physically existing formal systems. To maintain the physico-formalist thesis, it is worthwhile to illustrate that such an abstraction does not lead out of the realm of the physically existing formal systems.

Consider a number of different physically existing formal systems $L_{1}, L_{2}, \ldots L_{n}$, embodied in different particular physical systems (Fig. 2). To abstract from some peculiar properties of physical objects $L_{1}, L_{2}, \ldots L_{n}$, and to isolate the common essential features of them, first of all requires knowledge of the properties of the physical objects in question. That is, we must have a physical theory $(M, S)$-in the sense of the definition of physical theory in the next section-describing $L_{1}, L_{2}, L_{3}, \ldots L_{n}$, using another physically existing formal system $M$. Abstraction is a relationship, formulated in $(M, S)$, between two, a more detailed and a less detailed, representations of $L_{1}, L_{2}, L_{3}, \ldots L_{n}$. Only in a suitable formal system $M$ it is meaningful to talk about similarity or isomorphism between the structures describing $L_{1}, L_{2}, \ldots L_{n}$, and, for example, about the equivalence class of these structures, which could be regarded as the product of the abstraction process. But, all these are contained in $M$, a formal system existing in the physical world. Thus, abstraction does not lead out of the physical realm. It does not produce "abstract formal systems" over and above the physically existing ones. One physically embodied formal system can be applied in the description of another physically embodied formal system, that's all.

10. Thus, any statement about a formal system, including a statement like " $\Sigma \vdash A$ ", is a statement of a physical fact; consequently, it has exactly the same epistemological status as any other statements about the physical world. This has far-reaching consequences, of course: logical and mathematical truths express objective (mind independent) facts of a particular part of the physical world, namely, the facts of the formal systems themselves. As such, they are synthetic, a posteriori, not necessary, and not certain; they are fallible. But they have contingent factual content, as any similar scientific assertion, so they "can be true and useful and surprising" [5, p. 72]. The logical and mathematical facts can be discovered, like any other facts of nature, just like a fact about a plastic molecule, or other artifact. 
The fact that the formal systems usually are simple physical systems of relatively stable behavior, like a clockwork, and that the knowledge of logical and mathematical truths does not require observations of the physical world external to the formal systems explains the universal illusion that logical and mathematical truths are necessary, certain and a priori.

\section{Physical Theory}

11. A physical theory, providing a description of a certain part of physical reality, symbolically denoted by $U$, can be considered as a partially interpreted formal system. Symbolically I will denote such a theory by $(L, S)$, where $L$ is the formal system in question, and $S$ stands for the semantics of the theory, which we are going to define in the next point. The formal system $L$ is meant to include the language of the theory, the derivation patterns, and the axiom system which will be denoted below by $\Sigma_{L}$. Traditionally, the axioms are divided into the logical axioms, ${ }^{2}$ the axioms of some mathematical theories, and, of course, some physical axioms.

This approach goes back to Carnap's Theories as Partially Interpreted Formal Systems (1939):

Any physical theory, and likewise the whole physics, can in this way be presented in the form of an interpreted system, consisting of a specific calculus (axiom system) and a system of semantic rules for its interpretation; the axiom system is, tacitly or explicitly, based upon a logico-mathematical calculus with customary interpretation. [7, Section 23]

The account developed in the present paper may be taken in a way congruous with the original Carnapian view, but it differs in two significant ways. First, according to the (physico-)formalist approach, the "logico-mathematical calculus" in itself is just a formal system without "customary interpretation" whatsoever. Second, according to the conception of semantics presented in the next points, the link between the elements of the formal system and the physical world is not contained in the language of the theory, in the form of some correspondence rules. Rather, as will be seen, semantics is something partly external to the language of the theory; a phenomenon jointly produced by the formal system $L$ and the part of the external physical reality $U$ to be described.

12. The definition will be based on the intuition we can learn from Gödel's construction of representation of the meta-arithmetic statements in Peano arithmetic, in the preparation of the first incompleteness theorem (e.g. [8, pp. 52-54], [14, pp. 145146]). Gödel clearly defines, when we are entitled to say that a formula of a formal system (in his case, a formula of Peano arithmetic) represents/means/refers to a state of affairs of the world outside of the formal system (in his case, in meta-arithmetic). For example, consider the meta-arithmetic statement that 'the formula-sequence of

\footnotetext{
2 Although it is not important from the point of view of our present concern, it is worthwhile mentioning that there are strong arguments in favor of first-order logic, both in foundations of mathematics and in the axiomatization of physical theories (e.g. [2,4,31]).
} 
Gödel number $x$ constitutes a proof of the formula of Gödel number $y^{\prime}$, symbolized as $\operatorname{Pr}(x, y)$. Let $\{\operatorname{Pr}(x, y)\}_{x, y}$ denote the family of similar meta-arithmetic statements. For a given pair of Gödel numbers $x$ and $y, \operatorname{Pr}(x, y)$ can either be the case or not be the case. In Gödel's construction, finally, we have a corresponding family $\{R(\bar{x}, \bar{y})\}_{x, y}$ of formulas of PA, where $\bar{x}$ and $\bar{y}$ are the numerals corresponding to the Gödel numbers $x$ and $y$. The elements of $\{R(\bar{x}, \bar{y})\}_{x, y}$ represent the elements of $\{\operatorname{Pr}(x, y)\}_{x, y}$, specifically, $R(\bar{x}, \bar{y})$ represents $\operatorname{Pr}(x, y)$, due to the satisfaction of the following condition: for all Gödel numbers $x$ and $y$, that is, for all paired $\operatorname{Pr}(x, y)$ and $R(\bar{x}, \bar{y})$ from the two families,

$$
\begin{aligned}
& \text { if } \operatorname{Pr}(x, y) \text { is the case then } \Sigma_{\mathrm{PA}} \vdash R(\bar{x}, \bar{y}) \\
& \text { if } \operatorname{Pr}(x, y) \text { is not the case then } \Sigma_{\mathrm{PA}} \vdash \neg R(\bar{x}, \bar{y})
\end{aligned}
$$

where $\Sigma_{\mathrm{PA}}$ denotes the axioms of Peano arithmetic. The regularity that the above condition holds for the whole family $\{\operatorname{Pr}(x, y)\}_{x, y}$ of meta-arithmetic statements of a certain type is an essential ingredient of Gödel's conception of representation, and plays an important role in the proof of the theorem (e.g., [8, pp. 55-56]). For, for example, nothing would follow from assigning, by convention, one single true meta-arithmetic statement to one single theorem of PA. As a matter of fact, the metalanguage expression 'if . . then' in lines (1)-(2) would be meaningless if $\{\operatorname{Pr}(x, y)\}_{x, y}$ and $\{R(\bar{x}, \bar{y})\}_{x, y}$ had only one element.

Mutatis mutandis, a (partial) semantics for a formal system $L$ is given by

(A) a family $\left\{A_{\lambda}\right\}_{\lambda}$ of formulas in $L$ and a family $\left\{a_{\lambda}\right\}_{\lambda}$ of states of affairs in $U$, such that,

(B) for all $\lambda$, that is for all paired $a_{\lambda}$ and $A_{\lambda}$,

$$
\begin{aligned}
& \text { if } a_{\lambda} \text { is the case in } U \text { then } \Sigma_{L} \vdash A_{\lambda} \\
& \text { if } a_{\lambda} \text { is not the case in } U \text { then } \Sigma_{L} \vdash \neg A_{\lambda}
\end{aligned}
$$

In such way the formulas belonging to $\left\{A_{\lambda}\right\}_{\lambda}$ are endowed with meaning: $A_{\lambda}$ means or refers to $a_{\lambda}$.

13. A few important remarks are in order.

(a) As we have seen, to be a meaning-carrier is not simply a matter of convention or definition or declaration. Semantics is not an arbitrary assignment of states of affairs of the world to linguistic elements of the theory.

(b) It is pointless to talk about the meaning of an isolated formula of the theory. (Semantic holism) It is not only because a whole family of formulas are endowed with meaning, "as a corporate body", but also because in condition (B) a big part of the axiomatic system can be involved.

(c) It must be recognized that condition (B) is exactly the same as the necessary and sufficient condition for the theory $(L, S)$ to be true. That is, the two conceptions meaning and truth are completely intertwined.

(d) The semantics, in the above holistic sense, is a part and parcel of physical theory. In case of empirical failure of a physical theory, semantics is one of the possible 
object of revision. In other words, semantics is as much hypothetical as any other part of the theory.

14. It must be clear that $a_{\lambda}$, as a symbol in the meta-language we use to describe the semantic relationship, stands for a state of affairs, a configuration of the physical world. Also, according to the physico-formalist approach " $\Sigma_{L} \vdash A_{\lambda}$ " and " $\Sigma_{L} \vdash \neg A_{\lambda}^{\prime \prime}$, respectively, are states of affairs in the physical world, facts of the physically embodied formal system $L$. Thus, what we observe in condition (B) is a kind of regularity or correlation between physical facts of two parts of the physical world, $L$ and $U$. Combining this with the thesis of the principle of common cause, one must conclude that both semantic relationship and the truth of the physical theory (consequently, our knowledge) must be brought about by the underlying causal processes of the physical world, going on in the common causal past of the two parts of the world $L$ and $U$. This underlying process is what we normally call learning through experience. That is, no genuine knowledge of the physical world is possible without experience. By the same token, no semantically meaningful talk about the physical world is possible without experience. There is no a priori meaning and there is no a priori truth. ${ }^{3}$

15. Let us clarify then the role of logic and mathematics in our knowledge of the physical world. One might ask: if mathematics is only about the formal systems without meaning, how is it, then, possible that mathematical structures prove themselves to be so expressive in the physical applications? As Richard Feynman put it: "I find it quite amazing that it is possible to predict what will happen by mathematics, which is simply following rules which really have nothing to do with the original thing." [10, p. 171]

We need to separate the sociological/practical aspect of this issue from the epistemological one. Let me start with the sociological aspect. From sociological point of view, by "mathematics" we mean that part of the axioms of $L$ (see point 11) which is traditionally considered as non-physical axioms. It must be clear however that it is not "mathematics" alone by which the physicist can predict what will happen, but the physical axioms together with the logical and mathematical axioms. The physicist, keeping, as long as possible, the logical and mathematical axioms fixed, tunes the physical axioms such that the theorems derivable from the unified system of logical, mathematical, and physical axioms (together with the deduction rules) + the semantics, as a whole, be compatible with the empirical facts. Consequently, the employed logical and mathematical structures in themselves need not, and do not, reflect anything about the real world in order to be useful.

Let me recall an analogy I gave in [30]. You can experience a analogous situation when you change the mouse driver on your computer (or just change the mouse set-

\footnotetext{
3 The principle of common cause is the Reichenbachian thesis that no correlation without causation; every correlation is either due to a direct causal effect, or is brought about by a third factor, a so-called common cause [26]. Due to the difficulties encountered in the EPR-Bell problem, many argue that the principle fails in the realm of quantum mechanics. I do not wish to become embroiled, in this paper, in the debate as to whether the common cause principle, in precise formulation, deserves to be regarded as universally valid principle. My own view is that it does; none of the counter-examples provides a compelling reason to deny it (cf. $[3,15])$. In any event, learning from experience, as an existing and known physical process producing the correlation in question, is a natural causal explanation; no reason to say that this is a correlation without need of explanation, or to look for some mysterious alternative explanation like "pre-adaption" or "preestablished harmony" (also see point 15).
} 
tings): first you feel that the pointer movements ("derived theorems") generated by the new driver ("mathematics") according to your previously habituated hand movements ("physical axioms") do not faithfully reflect the arrangement of your screen content (experienced world). Then, keeping the driver (and driver settings) fixed, you tune your hand movements - through typical "trial and error" learning — such that the generated pointer movements fit to the arrangement of your screen content.

Thus, there is no miraculous "preadaption" or "pre-established harmony" involved just because certain aspects of empirical reality "fit themselves into the forms provided by mathematics". This is simply a result of the physicist's continuous choice from the store shelves of mathematics, and the continuous tuning the physical axioms.

From epistemological point of view the situation is even more simple. In a physical theory $(L, S)$, the formal system $L$ is a single undivided whole. As a formal system, $L$ can be the object of logical/mathematical investigation and knowledge. In itself, however, it has nothing to do with the physical world described by the physical theory $(L, S)$. In case of empirical failure of the physical theory $(L, S)$, any element of $L$ as well as the semantics can be the object of revision. Through this continuous "trial and error" process, the physicist tunes the physical theory $(L, S)$, as a whole, be compatible with the experienced world; satisfy condition (B) in point $\mathbf{1 2}$.

\section{Constitutive A Priori Reconsidered}

16. Consider the following example. Given that $L$ is consistent, one can easily see that the following statements cannot hold true at the same time:

(i) $A$ refers to $a$,

(ii) $\Sigma_{L} \vdash A$,

(iii) $a$ is not the case in $U$,

since (i) and (iii), according to condition (B) in point 12, would imply $\Sigma_{L} \vdash \neg A$, in contradiction with (ii). Therefore, observing that $a$ is not the case we are not entitled to say that we observe that " $\neg A$ ". Simply because if $a$ is not the case, then condition (B) fails, and the whole semantics is lost. Therefore $\neg A$ does not carry meaning at all. That is to say, we are not able to attribute a feature, whatsoever, to the physical reality in the situation when $a$ is not the case.

17. Let us denote this unexpressed, unarticulated state of affairs by $a^{*}$. Once a modified (or completely new) theory, $\left(L^{\prime}, S^{\prime}\right)$, is constructed with a new family of state of affairs $\left\{a_{\lambda^{\prime}}^{\prime}\right\}_{\lambda^{\prime}}$ and a new family of formulas $\left\{A_{\lambda^{\prime}}^{\prime}\right\}_{\lambda^{\prime}}$, such that $a^{*}=a_{\lambda_{*}^{\prime}}^{\prime}$ and condition (B) in point 12 is satisfied, the corresponding $A_{\lambda_{*}^{\prime}}^{\prime}$ will be attributed to $a^{*}$, as true feature of reality in state $a^{*}$.

18. This example not only confirms what was said in point $\mathbf{1 3}(\mathrm{d})$, but also sheds light on the constitutive role of semantics, similar to the constitutive role of Reichenbach's coordinative definitions we described in point $\mathbf{3}$. There are however significant differences:

(a) The whole account I developed remains within the clear and minimal ontological framework of physicalism. 
(b) All entities involved are contingently existing physical objects.

(c) Whatever is the concrete physical process (point 14) producing the correlation required in condition $(\mathrm{B})$ of point $\mathbf{1 2}$, that is, producing meaning and truth, it is a contingently existing part of the physical reality.

(d) Therefore, the whole "epistemic agency" involved is embodied in the physical world.

(e) The formal system in $(L, S)$ plays, indeed, a similar role as Reichenbach's "constitutive principle"; except that

- $L$ has a clear ontological status; it is a part of the physical world,

- all facts of $L$ are contingent facts of the physical world, therefore they do not generate necessarily true synthetic a priori statements about the physical world, for sure.

(f) There is no "conceptual side of the coordination". The constitutive role of formal systems by no means entitles us to say that there is a hypostatized a priori conceptual scheme in terms of which we grasp the experienced reality, and that this conceptual scheme generates analytic truths. For, what there is is anything but conceptual: we only have the physically existing formal systems which have no meaning. Once an otherwise meaningless formula of a formal system is provided with meaning, in the sense of point $\mathbf{1 2}$, it becomes true or false in a non-analytic sense.

(g) Certainly, semantics plays constitutive role; not in the form of isolated operational definitions of physical concepts, but in holistic sense. Moreover, semantics is completely intertwined with the truth of the theory; also, not in the form of the truth of isolated predictions, but in holistic sense of (B) in point $\mathbf{1 2 .}$

(h) In fact, therefore, what plays the constitutive role is the whole theory $(L, S)$, though, the whole theory is certainly not a priori. As we have seen, however, aprioricity is not required for playing a constitutive role. That is why there is no tension, whatsoever, between the fact that the applied logical and mathematical structures as well as the constructed semantics can change (see point 17) when a theory is superseded by another one, on the one hand, and their constitutive role in furnishing reality, on the other (cf. [12,17,23]).

(i) Finally, it is worthwhile to mention that the constitutive role of the theory $(L, S)$ in furnishing "the »continuum « of reality" [25, p. 50] with objects, properties, and relations, in itself, by no means implies the denial of realism; the belief that the existence of the objects, properties, and relations posited by the theory is a real feature of reality. What is true about scientific realism is also true about metaphysical realism - if the distinction is meaningful at all. For a "metaphysical" account must have the structure of $(L, S)$ in order to be meaningful and true, just in the sense we described in point $\mathbf{1 2}$.

19. Thus, the corrected formulation of the argument against intrinsic-extrinsic distinction, outlined in point $\mathbf{5}$, is the following. The features (attributes, qualities, properties) attributed to a real object are not possessed by the object as a "thing-in-itself"; they require the existence of something else: a physical theory $(L, S)$ by means of which these features are constituted. The existence of $(L, S)$ however implies the existence of 
a physically embodied formal system $L$ and a real causal process in the physical world producing the correlation required for both the semantics and the truth of the theory. All this means that a physical object can possess a property only if other contingently existing physical objects exist.

Acknowledgements Open access funding provided by Eötvös Loránd University (ELTE). Funding was provided by Hungarian National Research, Development and Innovation Office (Grant No. K115593).

Open Access This article is distributed under the terms of the Creative Commons Attribution 4.0 International License (http://creativecommons.org/licenses/by/4.0/), which permits unrestricted use, distribution, and reproduction in any medium, provided you give appropriate credit to the original author(s) and the source, provide a link to the Creative Commons license, and indicate if changes were made.

\section{References}

1. Allen, S.: Properties. The Internet Encyclopedia of Philosophy (2018). https://www.iep.utm.edu/ properties

2. Andréka, H., Németi, I., Madarász, J., Székely, G.: On Logical Analysis of Relativity Theories. arXiv:1105.0885 (2011)

3. Arntzenius, F.: Reichenbach's common cause principle. In: E.N. Zalta (ed.). https://plato.stanford.edu/ archives/fall2010/entries/physics-Rpcc/ (2010)

4. Ax, J.: The elementary foundations of spacetime. Found. Phys. 8, 507-546 (1978)

5. Ayer, A.J.: Language. Truth and Logic. Dover Publications, New York (1952)

6. Bell, E.T.: Mathematics: Queen and Servant of Science. McGraw-Hill Book Company, New York (1951)

7. Carnap, R.: Theories as Partially Interpreted Formal Systems. Foundations of Logic and Mathematics. University of Chicago Press, Chicago (1939)

8. Crossley, J.N., Ash, C.J., Stillwell, J.C., Williams, N.H., Brickhill, C.J.: What is Mathematical Logic?. Dover Publications, New York (1990)

9. Carr, D.: The Paradox of Subjectivity. The Self in the Transcendental Tradition. Oxford University Press, Oxford (1999)

10. Feynman, R.: The Character of Physical Law. MIT Press, Cambridge (1967)

11. Francescotti, R.: How to define intrinsic properties. Noûs 33, 590-609 (1999)

12. Friedman, M.: Dynamics of Reason. CSLI Publications, Stanford (2001)

13. Gömöri, M., Szabó, L.E.: Operational understanding of the covariance of classical electrodynamics. Phys. Essays 26, 361-370 (2013)

14. Hamilton, A.G.: Logic for Mathematicians. Cambridge University Press, Cambridge (1988)

15. Hofer-Szabó, G., Rédei, M., Szabó, L.E.: The Principle of the Common Cause. Cambridge University Press, Cambridge (2013)

16. Humberstone, L.: Intrinsic/extrinsic. Synthese 108, 205-267 (1996)

17. Ivanova, M.: Friedman's relativised a priori and structural realism. Search of compatibility. Int. Stud. Philos. Sci. 25, 23-37 (2011)

18. Langton, R., Lewis, D.: Defining 'intrinsic'. Philos. Phenomenol. Res. 58, 333-345 (1998)

19. Lewis, D.: Extrinsic properties. Philos. Stud. 44, 197-200 (1983)

20. Lewis, D.: Redefining 'Intrinsic'. Philos. Phenomenol. Res. 63, 381-398 (2001)

21. Marshall, D.: An analysis of intrinsicality. Noûs 50, 704-739 (2016)

22. Marshall, D., Weatherson, B.: Intrinsic vs. extrinsic properties.In: E.N. Zalta (ed.) The Stanford Encyclopedia of Philosophy (Spring 2018 Edition). https://plato.stanford.edu/archives/spr2018/entries/ intrinsic-extrinsic (2018)

23. McArthur, D.: Theory change, structural realism, and the relativised a priori. Int. Stud. Philos. Sci. 22, 5-20 (2008)

24. Quine, W.V.: Two Dogmas of empiricism. Philos. Rev. 60, 20-43 (1951)

25. Reichenbach, H.: The Theory of Relativity and a Priori Knowledge. University of California Press, Berkeley and Los Angeles (1965) 
26. Reichenbach, H.: The Direction of Time. University of California Press, Berkeley (1956)

27. Sider, T.: Intrinsic properties. Philos. Stud. 83, 1-27 (1996)

28. Szabó, L.E.: Formal system as physical objects: a physicalist account of mathematical truth. Int. Stud. Philos. Sci. 17, 117-125 (2003)

29. Szabó, L.E.: Mathematical facts in a physicalist ontology. Parallel Process. Lett. 22, 1240009 (2012)

30. Szabó, L.E.: Meaning, Truth, and Physics. In: Hofer-Szabó, G., Wroński, L. (eds.) Making it Formally Explicit. European Studies in Philosophy of Science, vol. 6. Springer, Berlin (2017)

31. Väänänen, J.: Second-order logic and foundations of mathematics. Bull. Symb. Logic 7, 504-520 (2001)

32. Vallentyne, P.: Intrinsic properties defined. Philos. Stud. 88, 209-219 (1997)

Publisher's Note Springer Nature remains neutral with regard to jurisdictional claims in published maps and institutional affiliations. 